\title{
WhatsApp: herramienta de comunicación educativa entre padres de familia y docentes de educación primaria
}

\author{
Fecha de recepción: 2021-09-27 • Fecha de aceptación: 2021-11-22 • Fecha de publicación: 2022-01-10
}

Gema María Loor Ramos ${ }^{1}$ Universidad Técnica de Manabí, Ecuador gloor8639@utm.edu.ec

https://orcid.org/0000-0003-4853-9649

Vicenta Inmaculada Aveiga Macay ${ }^{2}$ Universidad Técnica de Manabí, Ecuador vicenta.aveiga@utm.edu.ec

https://orcid.org/0000-0001-8028-7432

Walter José Zambrano Romero ${ }^{3}$ Universidad Técnica de Manabí, Ecuador walter.zambrano@utm.edu.ec https://orcid.org/0000-0002-0225-3955

\section{Resumen}

La educación en tiempos de Covid-19 es un reto en todas las instituciones educativas. El uso de las redes sociales se convirtió en el medio de comunicación idóneo para proporcionar información del que hacer académico a los padres de familia. El objetivo de esta investigación fue determinar el uso de la red social WhatsApp como herramienta de comunicación educativa entre docentes y padres de familia de la Unidad Educativa "Antonio José de Sucre Cedeño Cortez", del cantón 24 de mayo, Provincia de Manabí, Ecuador. En el desarrollo del estudio se aplicó la metodología mixta, con un enfoque cualitativo y cuantitativo, además del método analítico apoyado en el bibliográfico, mismos que permitieron recopilar los datos requeridos para el tema de investigación, con la aplicación de una encuesta a 13 padres de familia del cuarto año de Educación General Básica y 
la entrevista a 14 docentes de Educación Primaria. Los resultados revelaron que el 100\% de los padres de familia utilizan WhatsApp como herramienta de comunicación educativa, y el $71.4 \%$ de los docentes obtienen respuesta oportuna con el envío de actividades académicas y cualquier tipo de comunicación, por lo que se evidenció que esta red social constituye una estrategia de aprendizaje en los centros educativos.

Palabras clave: redes sociales, educación primaria, WhatsApp, Covid-19, profesores, padres de familia

\begin{abstract}
Education in times of Covid-19 is a challenge in all educational institutions. The use of social networks became the ideal means of communication to provide information on academic activities to parents. The objective of this research was to determine the use of the WhatsApp social network as a tool for educational communication between teachers and parents of the "Antonio José de Sucre Cedeño Cortez" Educational Unit, Canton 24 de Mayo, Province of Manabí, Ecuador. In the development of the study, a mixed methodology was applied, with a qualitative and quantitative approach, in addition to the analytical method supported by the bibliographic method, which allowed collecting the data required for the research topic, with the application of a survey to 13 parents of the fourth year of General Basic Education and the interview to 14 teachers of Elementary Education. The results revealed that $100 \%$ of parents use WhatsApp as an educational communication tool, and $71.4 \%$ of teachers get timely response with the sending of academic activities and any type of communication, so it was evidenced that this social network is a learning strategy in schools.
\end{abstract}




\section{Introducción}

La aparición de la pandemia del Covid-19 ha causado estragos en el mundo entero, a diversas escalas y en diferentes ámbitos, creando una serie de desafíos para los sistemas educativos a nivel mundial, obligando a todas las instituciones educativas a llevar a la práctica nuevos modelos de aprendizaje, por lo cual, se vieron "forzadas" a reinventarse durante este momento difícil, sacando de su zona de confort a los docentes, futuros educadores, padres de familia y a los mismos estudiantes (Nel \& Marais, 2020).

Es por ello que, en el ámbito de la educación, la pandemia obligó a las instituciones educativas a encontrar alternativas a los modelos tradicionales de aprendizaje, tales como la modalidad en línea, para continuar con el proceso educativo, lo que dio paso a este cambio abrupto de la forma tradicional de aprendizaje que ha presentado varios desafíos, debido a la falta de recursos tecnológicos (Molise \& Dube, 2020).

La investigación tiene como finalidad analizar la utilidad de la red social WhatsApp como herramienta de comunicación educativa entre docentes y padres de familia del cuarto año de educación general básica, en la Unidad Educativa Fiscal "Antonio José de Sucre Cedeño Cortez" del cantón 24 de mayo, Provincia de Manabí, Ecuador. Se pretende que con los resultados se pueda determinar la calidad de comunicación existente entre docentes y padres de familia, e inclusive datos como: redes sociales más utilizadas, nivel de conectividad, preferencia de dispositivos tecnológicos, y finalmente el nivel de estudio y ocupación laboral de los padres de familia.

En este sentido, las escuelas, estudiantes y familias están siendo impactadas por diferentes tipos de "divisiones digitales", primero está la versión de "la brecha digital", que describe el acceso reducido a Internet de banda ancha en áreas rurales y urbanas (Perrin, 2019). Sin embargo, desde el punto de vista de La Jornada (2020), a partir de la interrupción de las clases con modalidad presencial, aparece una brecha digital que se maximiza de manera especial, ya que existen estudiantes que no poseen habilidades de autopreparación y disciplina de manera especial, por otra parte, existen otros que no cuentan con conexión a Internet o equipos de cómputo en sus hogares.

No obstante, un estudio realizado por WeAreSocial y Hootsuite en Ecuador, indican que existen 10,17 millones de usuarios conectados a Internet, cuya penetración se situó en el 57,3\%, por otro lado, el uso de redes sociales es de 14,00 millones de usuarios que equivalen al $78,8 \%$ de la población total hasta enero de 2021, además, el uso de celulares en Ecuador es de 13,82 millones de conexiones móviles que equivalen al $77,8 \%$ de la población total (Datareportal, 2021), como se muestra en la Figura 1. 


\section{Figura 1.}

Estadísticas de Internet, redes sociales y dispositivos móviles en Ecuador

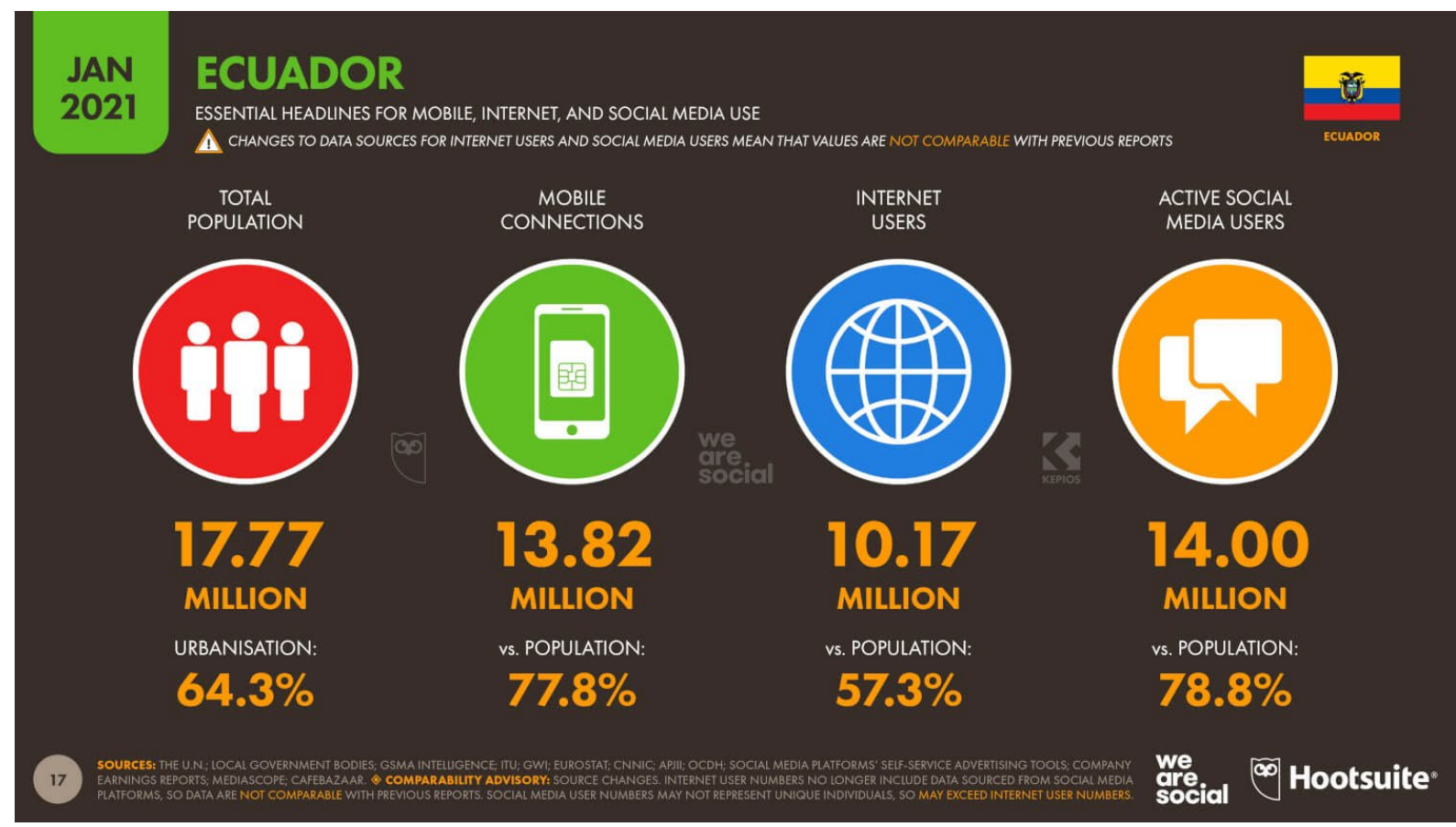

Fuente: Datareportal (2021)

Además, en el 2019 las redes sociales más utilizadas por la población en las áreas urbana y rural fueron: WhatsApp y Facebook, con el 63,8\% (población urbana) y 36,7\% (población rural) usan Facebook, mientras que, el 61,4\% (población urbana) y 30,8\% (población rural) usan WhatsApp (INEC, 2019), como se evidencia en la Figura 2. 
Figura 2.

Perfil de usuarios de redes sociales

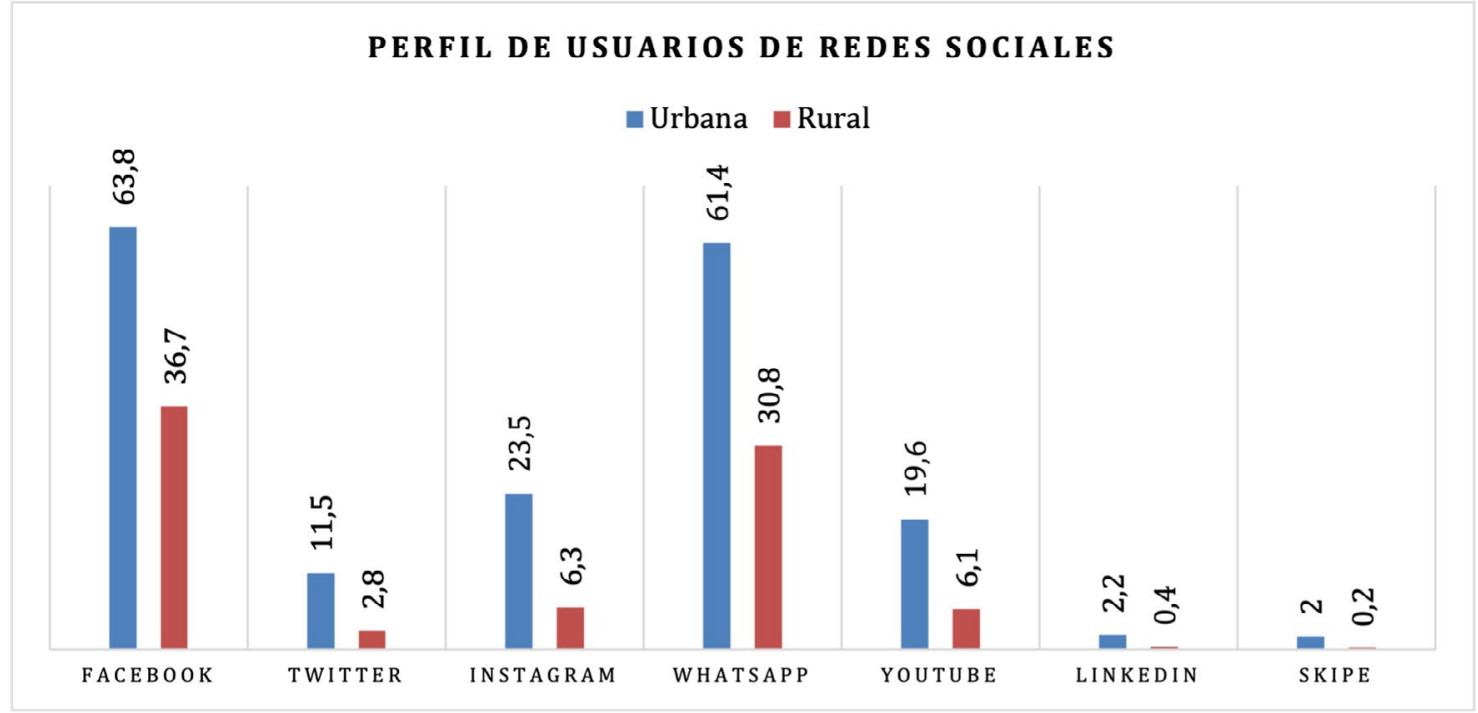

En ese contexto, las nuevas tecnologías de la información son precisamente uno de los canales de socialización con más alcance en la actualidad, además, fomentan nuevas formas de establecer relaciones sociales, por lo que se han convertido en un soporte elemental en el proceso educativo, debido a que facilitan el estudio acorde a las necesidades vigentes, y permiten desarrollar una conducta analítica sobre la realidad, basada en tecnologías. Pero debido al masivo uso de las TIC, y su progresiva evolución, los docentes se ven forzados a adquirir nuevas competencias digitales, las cuales deben ser estudiadas, profundizadas y actualizadas continuamente, con el objetivo de aplicarlas eficazmente en el proceso de enseñanza-aprendizaje (Díaz et al., 2021).

Asimismo, los estudios han demostrado que simplemente agregar una variedad de herramientas digitales dentro de los programas de estudio no es lo suficiente para que el trabajo de los docentes sea eficaz, lo que realmente marca la diferencia es el pensamiento reflexivo e intencional y la integración de tales herramientas dentro de comunidades y/o redes de profesionales de práctica (Cifuentes et al., 2011).

Por ende, hoy en día las familias tienen un rol trascendental en la educación de sus hijos e hijas por cuanto, tienen mayor protagonismo a la hora de ser su guía educativa. Autores como Pearson (2020), en sus estudios realizados, detecta que en Sudáfrica la mayoría de las escuelas no estaban preparadas para la enseñanza en línea, por lo tanto, los maestros y administradores se inscribieron frenéticamente en cursos para encontrar formas de garantizar que pudieran ayudar a sus estudiantes durante este tiempo.

Desde esta perspectiva, es fundamental resaltar el análisis de Mawarni et al. (2020), quienes en su investigación manifiestan que el aprendizaje en línea no se debe aplicar sin antes haber una competencia digital bien establecida, por cuanto, el éxito del proceso de aprendizaje dependerá de la preparación y motivación de los estudiantes y del apoyo de los padres de familia. Por otro 
lado, en el ámbito digital, la red social es un sitio web donde las personas pueden generar un perfil social, compartir información, participar en la generación de contenidos y colaborar en tendencias sociales (Cascales-Martínez et al., 2020).

Con respecto a lo anterior, una red social simula una vida virtual, donde su principal recurso es la información personal de sus usuarios, y tiene como objetivo compartir información y entablar relaciones humanas. Es por ello que, para asegurar que el aprendizaje se lleve a cabo en medio de desafíos, las instituciones educativas recurrieron al uso de WhatsApp como una herramienta de comunicación educativa y como una plataforma formal de enseñanza y aprendizaje, la cual permite utilizarla dentro y fuera del aula para alcanzar objetivos pedagógicos y de seguimiento de los estudiantes (Motaung \& Dube, 2020).

Es importante recalcar que Pan et al. (2015), en concordancia con Gannon \& Prothero, (2018), manifiestan que, los sitios y aplicaciones de redes sociales incluido WhatsApp se han integrado en comunidades, facilitando así lo que se llama una "comunidad virtual de práctica", dado a la fluidez y efectividad en la comunicación, el acceso e intercambio de información en cualquier momento y desde cualquier lugar, la posibilidad de enviar diferentes archivos digitales, documentos de textos, fotografías, enlaces, vídeos, etc, que son las características principales que han convertido a WhatsApp en una herramienta eficiente para el sector de la educación.

Por consiguiente, muchas escuelas adoptaron Zoom y Google Classroom, mientras que el departamento de educación básica inició varias lecciones de radio y televisión (Brodie et al., 2020). Bajo ese punto de vista como país, a Ecuador le faltó un plan de capacitación más efectivo en herramientas digitales y material de trabajo para preparar a los docentes en la educación en línea y la realización de un diseño instruccional para esta nueva modalidad de estudio, la que significó un verdadero reto tanto para el docente, el padre de familia y el estudiante. En ese sentido, la familia y la escuela son dos contextos próximos en aportan a la experiencia diaria de los estudiantes; es decir, comparten compromisos que involucran una comunicación y participación coherente, todo con la finalidad de potenciar el crecimiento físico, psicológico y social de los estudiantes (Rodríguez et al., 2019).

Para Rubio-Romero et al. (2019), la integración de las redes sociales influye en la educación en el actual mundo virtualizado y está apoyado esencialmente desde la aparición de los Smartphone, ya que las personas, sin importar la edad, están familiarizados con espacios virtuales como WhatsApp, YouTube, Facebook e Instagram. El mundo virtual se manifiesta a los adolescentes como alternativa a la vida real, y ellos acceden a él para realizar sociabilidad cuando se encuentran sin oficio. Según Cascales-Martínez et al. (2020), las redes sociales generan un excesivo interés, debido a que aportan al desarrollo de nuevas dinámicas sociales, relaciones humanas para todas las edades, tal es el caso del uso de WhatsApp, cuya aplicación se ha convertido en un recurso educativo.

Resulta oportuno mencionar lo expuesto por las autoras Nuuyoma et al. (2020), quienes explican que esta es una aplicación de mensajería instantánea cifrada, la misma que es compatible con teléfonos inteligentes y que, está diseñada para enviar, recibir mensajes de texto, imágenes, mensajes de audio, videos, documentos, ubicaciones de usuarios y contactos a través de Internet. 
En ese sentido, se evidencia que esta forma de comunicación va en auge, siendo una vía de comunicación de carácter más interpersonal o directo, en este caso, la red social WhatsApp como herramienta de comunicación entre padres de familia y docentes de entidades educativas, además, que las redes sociales son el fruto de un advenimiento tecnológico y de nuevos entornos digitales que modifican la sociedad de forma continua.

En ese contexto, la unidad educativa "Antonio José de Sucre Cedeño Cortez" es un centro educativo de educación regular y sostenimiento fiscal, con jurisdicción hispana, con código AMIE: 13H03766, la cual ofrece niveles educativos como educación general básica (EGB) y bachillerato, con modalidad presencial y jornada vespertina, localizada geográficamente en Manabí en el Cantón 24 de mayo de la Parroquia Bellavista.

Tras la pandemia Covid-19, el confinamiento y el cierre de las unidades educativas puesto que, eran considerados como focos potenciales de transmisión masiva del virus se enfrentaron al desafío de la educación virtual, convirtiendo las redes sociales en herramientas indispensables para el proceso educativo, es por ello que la unidad educativa anteriormente mencionada utilizó la red social WhatsApp como principal recurso educativo, con lo que mediante la creación de grupos permitió mantener comunicación con padres de familia y estudiantes.

\section{Metodología}

El estudio se realizó en la unidad educativa "Antonio José de Sucre Cedeño Cortez", perteneciente al cantón 24 de mayo, Provincia de Manabí con una población total de 13 padres de familia, representantes de 32 estudiantes, puesto que cada uno tiene más de un representado. Se escogió el cuarto año de educación general básica como parte de un plan piloto de los siete niveles que tiene la institución, siendo este el que tiene mayor número de estudiantes, además, la ubicación de los hogares de los encuestados son los más cercanos, al ser una escuela de la zona rural, por lo tanto, en caso de que existieran inconvenientes en llenar la encuesta, se realizaba visitas in situ, por otro lado, no todos los padres de familia de los niños y niñas cuentan con las competencias adecuadas para manipular un dispositivo tecnológico, en relación a los profesores se entrevistó a todos (14) los que corresponden al nivel primario de la unidad educativa en estudio.

La investigación fue de tipo descriptiva, sistemática y también posee enfoque cuantitativo, en primera instancia se efectuó una revisión en bases de datos certificadas sobre diversos conceptos emitidos por autores en relevancia al tema propuesto, lo que permitió dar sustento teórico, luego se aplicó la técnica de la entrevista a los docentes de educación primaria de la unidad educativa, a través de la plataforma Google Meets y consecuentemente una encuesta mediante un formulario de Google Form a los padres de familia, la cual se procedió a enviar el link mediante el grupo de WhatsApp, finalmente, para la tabulación de datos se usó Microsoft Excel.

Además, el método analítico utilizado fue el modelo interactivo de Miles et al. (2018) que consta de 4 etapas, tales como; recopilar datos, formular datos, presentar datos y dibujar conclusiones (Mawarni et al. 2020), por otro lado, el levantamiento de datos preliminares permitió conocer la condición socio económica y el contexto histórico concreto de su residencia. 
Los instrumentos utilizados para la obtención de datos fueron un cuestionario para la entrevista compuesto por siete (7) preguntas, el cual fue dirigido a los docentes, y otro cuestionario hecho en Google Form, para encuestar a los padres, que estuvo compuesto por nueve (9) preguntas, con el objetivo de conocer la eficacia de WhatsApp como herramienta de comunicación entre docentes y padres de familia, durante la pandemia de Covid-19, es por ello que, una vez desarrollado y revisado el instrumento para la obtención de información, se realizó un acercamiento al centro educativo para solicitar la autorización para aplicar la entrevista.

Para la ejecución de los instrumentos primero se construyó el formulario en Google, luego se procedió a enviar la encuesta a los 13 padres de familia al grupo de WhatsApp del cuarto año de educación básica, el instrumento fue desarrollado en base a preguntas abiertas y cerradas, tanto para la entrevista, como para la encuesta.

\section{Resultados}

Los resultados obtenidos en este artículo fueron analizados de forma objetiva. A continuación, se presenta el análisis extraído de las entrevistas aplicadas a los docentes y de la misma manera la encuesta implementada a los padres de familia.

\subsection{Revisión e interpretación de resultados acorde a la entrevista realizada a los docentes}

La aplicación de la entrevista se realizó a 14 docentes de primero a séptimo año de educación primaria, la primera pregunta de la guía estaba direccionada a conocer si recibieron capacitaciones por parte del Ministerio de Educación, donde el 100\% de los docentes entrevistados manifestaron que sí recibieron capacitaciones. Por lo que resulta ventajoso para ellos, a la hora de manipular las aplicaciones dictaminadas por el organismo, donde ellos contestaron que fueron Microsoft Teams, Moodle, Edmodo. Pero lamentablemente comentan de manera general, que los estudiantes y padres de familia no tienen conocimientos en herramientas digitales educativas, como las mencionadas anteriormente. Por lo que conviene utilizar herramientas más accesibles y fáciles de manipular, en este caso WhatsApp o Facebook.

La segunda pregunta estaba relacionada para conocer si como docentes, consideran a la red social WhatsApp como un recurso educativo y un medio idóneo para mantener informados a los padres de familia en el proceso educativo, en la etapa de educación primaria durante la pandemia Covid-19, de los 14 docentes entrevistados, 13 respondieron que sí consideran la red social WhatsApp como un recurso educativo y un medio idóneo para compartir información sobre el proceso educativo, lo que equivale al $92.9 \%$, mientras que uno de los entrevistados respondió que no considera a la red social como recurso educativo, ni como un medio idóneo para mantener comunicación con los padres de familia.

Otra de las interrogantes estaba orientada en detectar cuáles fueron las experiencias y obstáculos que experimentaron al utilizar WhatsApp durante el proceso educativo en la Pandemia Covid-19, donde los entrevistados resaltaron que fue satisfactoria porque les permitió estar en constante comunicación, tanto con los padres de familia, como con los estudiantes, y la red social WhatsApp es una herramienta de fácil aplicación y accesible de manejar, la misma que está al alcance de 
todos, por otro lado, permite compartir audios, videos, realizar llamadas y videollamadas, lo que facilita la comunicación entre los miembros de la comunidad educativa.

Sobre los obstáculos presentados, los docentes entrevistados mencionaron que la falta de conectividad e Internet fija en ciertos hogares, en algunos caso una cobertura no muy buena, o la falta de dispositivos móviles actualizados fueron las dificultades más comunes a las que se enfrentan diariamente, es por ello que, como política de estado, es necesario implementar el servicio de Internet gratuito en las zonas rurales y con ello los estudiantes puedan recibir clases sincrónicas, que es una solicitud de los padres de familia y así puedan interactuar con el docente y mejorar la calidad de la educación.

Seguido a ello, otra de las preguntas estaba relacionada en qué tan efectivo consideran el uso del WhatsApp en el proceso de enseñanza-aprendizaje para sus estudiantes, de los 14 docentes entrevistados, 9 de ellos, que corresponde al 64.3\%, manifestaron que consideran muy bueno el uso de WhatsApp en el proceso de enseñanza-aprendizaje, porque otorga simplicidad y velocidad en la transmisión de información, otros 5 docentes, equivalente al 35.7\%, afirman que es muy intuitivo y directo a la hora de comunicar, revisar y enviar recursos óptimos (PDF, videos, audios, entre otros), para el proceso de aprendizaje.

Por otra parte, la sexta interrogante hacía referencia a si obtienen respuesta oportuna por parte de los padres de familia cuando se les emite algún comunicado, de los 14 docentes entrevistados, 10 de ellos respondieron que sí obtienen respuesta oportuna por parte de los padres de familia cuando se emite algún tipo de comunicación, lo que corresponde al $71.4 \%$, 3 manifestaron que a veces, lo que equivale al $21.4 \%$, y uno expresó que no obtiene respuesta oportuna cuando emite algún comunicado.

En la última pregunta se solicitó el punto de vista de cada uno de ellos de acuerdo a sus experiencias en esta nueva modalidad de estudios y qué recomendaciones podrían hacer al Ministerio de Educación para el próximo periodo lectivo, en sus respuestas mencionaron que se provea de Internet a las familias o se gestione para mejorar la cobertura en la zona rural de 24 de Mayo, para poder dar clases sincrónicas, otra de las sugerencias es procurar que la población estudiantil y las instituciones educativas posean laboratorios dotados con equipos informáticos y una buena conexión de Internet, para que de esta forma los estudiantes que no poseen esta tecnología tengan un lugar seguro y un punto fijo donde puedan acercarse a realizar sus actividades.

Considerando la experiencia de ellos, expresaron que la pandemia abrió los ojos y exigió a utilizar constantemente la tecnología del siglo XXI y es lo mejor que puede implementarse para los años posteriores, y de tal manera, capacitar a los docentes en lo que respecta al dominio de las TIC, además, los profesores sugieren incorporar la asignatura de computación a partir de cuarto año de educación general básica, a través de la modalidad de trabajo más eficaz para el proceso de su educación y de acuerdo a las circunstancias, implementar clases presenciales, considerando las medidas de bioseguridad de toda la comunidad educativa, para evitar el aumento en el nivel de contagios. 


\subsection{Revisión e interpretación de resultados acorde a la encuesta aplicada a los padres de familia}

En la Figura 3 se observan los resultados obtenidos sobre la conectividad, de los cuales nueve padres de familias, que corresponden al $69.2 \%$, manifestaron que si cuentan con conectividad fija de Internet y cuatro de ellos, que equivale a un $30.8 \%$, expresaron que utilizan recargas para descargar las tareas que envían los docentes.

Figura 3.

Conectividad de los padres en sus hogares

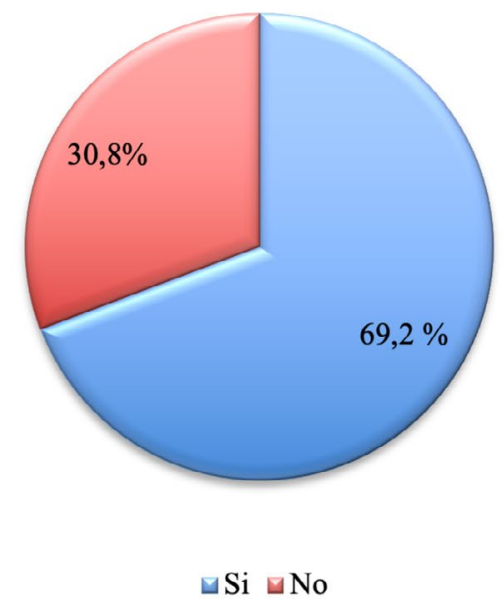

En lo que respecta a los dispositivos inteligentes con lo que cuentan en el hogar, el celular es el dispositivo más utilizado con el que se conectan en todos los hogares encuestado, lo que representa el $100 \%$, de los cuales siete hogares cuentan con un celular y seis hogares cuentan con dos celulares, estos resultados permiten conocer que la mayoría de los padres de familia tienen como medio de comunicación un dispositivo móvil, ninguno de los hogares de los encuestados cuentan con tablet, dos familias cuenta con una computadora de escritorio y tres hogares con una laptop.

Aunado en ello, los resultados sobre el tipo de dispositivos inteligentes que utilizan los estudiantes para recibir sus actividades escolares, donde el $100 \%$ de los encuestados manifestaron que el celular es el dispositivo móvil que utilizan para conectarse y mantenerse informados sobres convocatorias y diferentes actividades educativas.

En cuanto al uso de dispositivos inteligentes, se les interrogó si cada estudiante cuenta con un aparato tecnológico para realizar sus actividades escolares, donde los resultados mostraron que el $84.6 \%$ de los niños no cuentan con su propio dispositivo inteligente, por lo tanto, comparten el dispositivo para estudiar y el $15.4 \%$ si cuenta con un dispositivo por estudiante, tal como se ve en la Figura 4. 
Figura 4.

Dispositivo inteligente para comunicarse

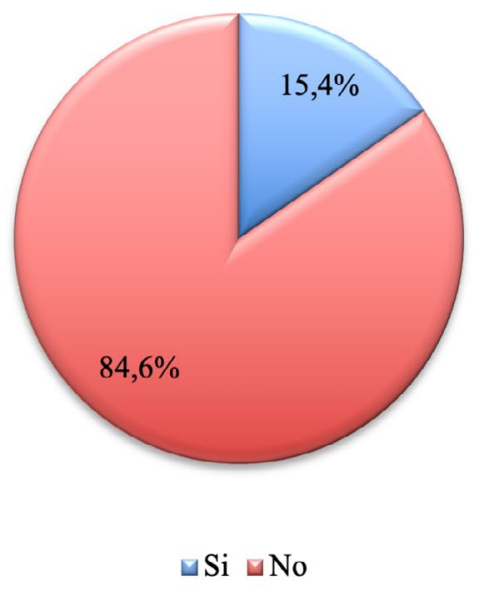

En lo que concierne, a las redes sociales utilizadas con mayor frecuencia por los padres de familia, la de mayor uso es WhatsApp, lo que equivale al 100\% de los encuestados, permitiendo corroborar que es la red social más recurrente, esto se debe a que es una herramienta accesible y de fácil manejo, que proporciona una comunicación rápida y directa, es por ello que, los padres de familia por medio de la red social WhatsApp están en comunicación constante con los docentes.

También es importante indagar sobre la velocidad de comunicación que ofrece WhatsApp, a lo que el $100 \%$ de los encuestados y entrevistados manifestaron que se puede mantener una comunicación muy fluida y asertiva.

Por otro lado, se consultó el último grado de estudio de los padres de familia, donde el $84,6 \%$ (Figura 5), que corresponde a ocho padres, terminaron el bachillerato, de los cuales seis padres terminaron completamente el nivel secundario, dos lo dejaron incompleto, y el 15,4\% que equivale a cinco padres de familia tienen instrucción primaria, donde dos de los entrevistados terminaron la primaria completa y tres con nivel incompleto, es por ello que, los padres de familia que no lograron terminar la primaria o los que presentan dificultades en la comprensión de las actividades escolares, indicaron que se ayudan con familiares para la realización de las tareas con sus hijos. 
Figura 5.

Nivel de estudio de los padres de familia

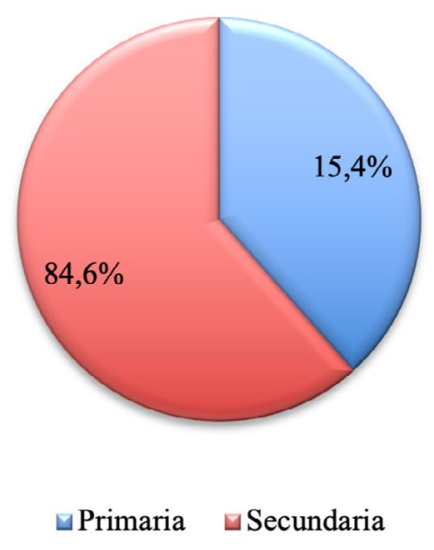

En la siguiente Figura 6 se muestran los resultados sobre la ocupación de los padres de familia, donde el $92.3 \%$ que corresponde a doce de los padres de familia son ejecutivas del hogar y el $7.7 \%$, que equivale a uno, estudia una carrera universitaria en línea, por lo tanto, se puede corroborar que los estudiantes cuentan con el apoyo de sus padres o demás familiares para el desarrollo de sus actividades escolares.

Figura 6.

Ocupación de los padres de familia

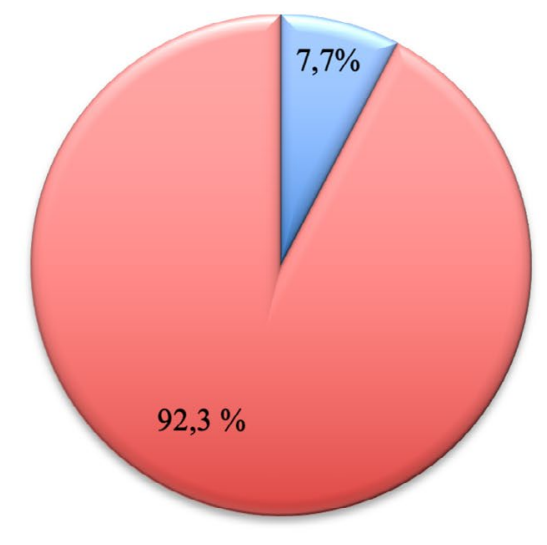

$\square$ Estudia $\square$ Ejecutiva del hogar $\square$ Trabaja

Finalmente, en la última pregunta se solicitó el punto de vista de cada uno de los entrevistados, ¿Qué medidas debería aplicar el Ministerio de Educación para mejorar la educación de los niños 
en tiempos de pandemia? En la que ellos respondieron, teniendo una buena conectividad, además, es indispensable que el Gobierno ecuatoriano implemente Internet en zonas rurales, para que los alumnos puedan cumplir con la realización de las diferentes actividades educativas que emiten los profesores, y así los docentes se sientan tranquilos a la hora de impartir sus clases, y aún más los estudiantes que puedan recibir tales conocimientos, mencionaron de manera adicional que el Gobierno debería dotar con recursos tecnológicos a las escuelas para que internamente ellas sean las encargadas de poner en disposición tales equipos a los niños de escasos recursos económicos.

\section{Discusión}

Los resultados obtenidos en esta investigación son coherentes con los hallazgos realizados por Maphosa et al. (2020) en la Universidad Estatal de Lupane, estos señalaron que el $77 \%$ de los estudiantes declaró que WhatsApp les ofreció oportunidades de aprender incluso durante la pandemia de COVID-19, además, revelaron que el $80 \%$ de los estudiantes coincidió en que la herramienta era fácil de maniobrar.

Según Paredes \& Zambrano (2020), en su investigación sobre las estrategias educativas que ofrece WhatsApp dentro de la Unidad Educativa 5 de junio de la ciudad de Manta, Manabí, Ecuador, definieron que WhatsApp es una aplicación usada por las personas, en este caso estudiantes, para realizar actividades académicas en el hogar y para otros fines como la comunicación social; considerando como un aspecto positivo la comunicación efectiva entre docentes, estudiantes y padres de familia.

Al respecto, Mawarni et al. (2020), manifiestan que es importante mencionar que el docente de la asignatura considere que, al usar WhatsApp, los estudiantes pueden acceder a él más fácilmente, puesto que ni siquiera requiere de muchas cuotas de paquetes de Internet, al realizar el aprendizaje en línea; sin embargo, los sujetos del sistema digital nunca han utilizado WhatsApp durante el proceso de aprendizaje, lo que se convierte en todo un reto en la educación.

En este sentido, el surgimiento de las redes sociales y aplicaciones informáticas han generado una evolución en las prácticas sociales, ya que plantean desafíos (García \& Cantón, 2019), lo que exige a los maestros profundizar en la alfabetización digital para contribuir a la innovación de procesos, la mejora del aprendizaje y la comunicación entre la familia y la institución educativa. Los acontecimientos anteriores permiten la aparición de nuevas líneas de investigación en la formación pedagógica, y esto a su vez se debe al uso de nuevas herramientas de comunicación (Cascales-Martínez et al., 2020).

Continuando con este análisis, resulta oportuno mencionar los estudios realizado por Basitere \& Mapatagane (2018) sobre el impacto de las redes sociales, particularmente WhatsApp en la participación y colaboración de los estudiantes, quienes determinaron que WhatsApp fue útil para mantener la interacción y la cooperación de los estudiantes durante el aprendizaje en temas de discusión y foros. 
Por su parte, Alshayeb (2018), en su estudio, encuentra que el uso de las redes sociales incursiona al estudiante a aprender autónomamente a medida que va formando estructuras de relaciones sociales. Por consiguiente, es imperante la necesidad de usar las herramientas digitales en la educación; sin embargo, Alva de la Selva (2015) y Zevallos et al. (2020) manifiestan la presencia de brechas digitales en sus múltiples facetas en América Latina, donde es indispensable abordar en la desigualdad existente a la hora que un estudiante cuente con un dispositivo electrónico o Internet en tales casos, por lo tanto, las instituciones educativas deben tener un modelo de madurez y transformación digital sin importar la locación donde se encuentre. De esta manera se podría evitar que se amplíen las desigualdades en el aprendizaje, favoreciendo a la igualdad de oportunidades en el ámbito de la educación (Giannini, 2020).

\section{Conclusiones}

La herramienta WhatsApp posibilitó una estrecha relación entre los padres de familia y docentes de la unidad educativa "Antonio José de Sucre Cedeño Cortez" del cantón 24 de mayo, Provincia de Manabí, durante la pandemia de COVID-19.

Se determina que WhatsApp es una herramienta de comunicación educativa adecuada en contextos de educación primaria, por cuanto proporciona una comunicación asertiva entre padres de familias y docentes de la institución en el proceso de enseñanza- aprendizaje.

La implementación de esta red social, como herramienta de comunicación educativa entre padres de familia y docentes de la Unidad Educativa "Antonio José de Sucre Cedeño Cortez" del cantón 24 de mayo, Provincia de Manabí, resultó exitosa de acuerdo a los resultados obtenidos. 


\section{Referencias}

Alshayeb, M. (2018). Promoting student engagement using social media technologies. In 6th International Conference of Advanced Computer Science \& Information Technology, 96-105. https://doi.org/10.5121/ csit.2018.80807

Alva de la Selva, A. (2015). Los nuevos rostros de la desigualdad en el Siglo XXI: la brecha digital. Revista Mexicana de Ciencias Políticas y Sociales, 60(223), 265-285. https://doi.org/10.1016/S0185-1918(15)72138-0

Basitere, M., \& Mapatagane, N. (2018, June). Effects of a Social Media Network Site on Student's Engagement and Collaboration: A case study of WhatsApp at a University of Technology. In ECSM 2018 5th European conference on social media.

Brodie, M., McFarlane, R., \& Nurina, A. (21 de abril de 2020). Learning in the time of Covid-19: Equitable support during school closures desperately needed. Daily Maverick. https://www.dailymaverick.co.za/ article/2020-04-21-learning-in-the-time-of-covid-19-equitable-support-during-school-closures-desperately-needed/\#gsc.tab=0

Cascales-Martínez, A., Gomariz, M.A, \& Paco, A. (2020). WhatsApp como herramienta educativa en Educación Primaria: alumnado, docentes y familias. Píxel-Bit: Revista de Medios y Educación, (58), 71-89. https:// doi.org/10.12795/pixelbit.74213

Cifuentes, L. Maxwell, G. \& Bulu, S. (2011). Technology Integration through Professional Learning Community. Journal of Educational Computing Research, 44(1), 59-82. https://doi.org/10.2190/EC.44.1.d

Datareportal. (11 de febrero de 2021). Digital 2021: Ecuador. https://datareportal.com/reports/digital-2021-ecua$\underline{\text { dor }}$

Díaz Vera, J. P., Ruiz Ramírez, A. K., \& Egüez Cevallos, C. (2021). Impacto de las TIC: desafíos y oportunidades de la Educación Superior frente al COVID-19. Revista Científica UISRAEL, 8(2), 113-134. https://doi. org/10.35290/rcui.v8n2.2021.448

Gannon, V. \& Prothero, A. (2018). Beauty bloggers and Youtubers as a community of practice. Journal of Marketing Management, 34(7 8), 592 619. https://doi.org/10.1080/0267257X.2018.1482941

García, S. \& Cantón, I. (2019). Teachers 3.0: Patterns of use of five digital tools. Digital Education, (35), $202-215$. https://revistes.ub.edu/index.php/der/article/view/26910/pdf

Giannini, S. (2020). COVID-19 y educación superior: De los efectos inmediatos al día después. Revista Latinoamericana de Educación Comparada, 11(17), 1-57. 
Instituto Nacional de Estadística y Censos. (2019). Encuesta Multipropósito - TIC 2019. INEC. https://www. ecuadorencifras.gob.ec/documentos/web-inec/Estadisticas Sociales/TIC/2019/201912 Principales resultados Multiproposito TIC.pdf

La Jornada. (31 de marzo de 2020). Clases digitales marcarán una brecha de aprendizaje: Schmelkes. https:// www.jornada.com.mx/ultimas/sociedad/2020/03/31/clases-digitales-marcaran-una-brecha-de-aprendizaje-schmelkes-5346.html

Maphosa, V., Dube, B. \& Jita, T. (2020). A UTAUT Evaluation of WhatsApp as a Tool for Lecture Delivery during the COVID-19 Lockdown at a Zimbabwean University. International Journal of Higher Education, 9(5), 8493. https://doi.org/10.5430/ijhe.v9n5p84

Mawarni, I., Ratnasari, N., Handayani, A. N., Muladi, M., Wibowo, E., \& Untari, R. (2020). Effectiveness of Whatsapp in improving student learning interests during the Covid-19 pandemic. In 2020 4th International Conference on Vocational Education and Training (ICOVET), 248-252. IEEE. https://ieeexplore.ieee.org/ stamp/stamp.jsp?tp=\&arnumber=9230031

Miles, M., Huberman, A., \& Saldaña, J. (2018). Qualitative data analysis: A methods sourcebook. Sage publications.

Molise, H., \& Dube, B. (2020). Emergency online teaching in Economic and Management Sciences necessitated by the COVID-19 pandemic: The Need for Healthy Relations in a Rural Schooling Context. International Journal of Learning, Teaching and Educational Research, 19(6), 387-400. https://doi.org/10.26803/ ijlter.19.6.23

Motaung, L., \& Dube, B. (2020). WhatsApp Messenger as a Mediating Tool in Times of COVID-19 for Enhancing Student Engagement in e-Tutorials at a Rural South African University. Journal of Educational and Social Research, 10(6), 214-214. https://doi.org/10.36941/jesr-2020-0121

Nel, C., \& Marais, E. (2020). Preservice teachers use of WhatsApp to explain subject content to school children during the COVID-19 pandemic. International Journal of Work-Integrated Learning, 21(5), 629-630. https:// doi.org/10.1016/S1470-2045(20)30217-5

Nuuyoma, V., Mhlope, N. \& Chihururu, L. (2020). The Use of WhatsApp as An Educational Communication Tool in Higher Education: Experiences of Nursing Students in Kavango East, Namibia. International Journal of Higher Education, 9(5). https://doi.org/10.5430/ijhe.v9n5p105

Pan, Y., Xu, Y., Wang, X., Zhang, C., Ling, H. \& Lin, J. (2015). Integrating social networking support for dyadic knowledge exchange: A study in a virtual community of practice. Information and Management, 52(1), 61 70. https://doi.org/10.1016/j.im.2014.10.001 
Paredes, B., \& Zambrano, L. (2020). Procedimientos formativos innovadores para la promoción del aprendizaje colaborativo en la aplicación de mensajería instantánea

WhatsApp. Rehuso, 5(1), 60-68. https://revistas.utm.edu.ec/index.php/Rehuso/article/view/1684

Pearson, S. (2020). How we are helping education in South Africa for those affected by the Coronavirus (COVID-19).

Perrin, A. (03 de junio de 2019). Digital gap between rural and nonrural America persists. Pew Research Center. https://www.benton.org/headlines/digital-gap-between-rural-and-nonrural-america-persists-0

Rodríguez, L. A., Escobar, M. C., Aveiga, V. I., \& Durán, U. C. (2019). Estrategia de Formación y Desarrollo de la Competencia Docente Ilamada Gestionar la Orientación Educativa Familiar, en la Educación Básica Superior. Información tecnológica, 30(6), 277-288. https://doi.org/10.4067/S0718-07642019000600277

Rubio-Romero, J., Jiménez, J. M., \& Barón-Dulce, G. (2019). Las redes sociales digitales como espacios de sociabilidad de los adolescentes. El caso del colegio Escolapios de Aluche. Revista Mediterránea de Comunicación, 10(2). https://doi.org/10.14198/MEDCOM2019.10.2.19

Zevallos, A., Martel, C., Pajuelo, P., Chávez, J., \& Palma, D. (2021). Perfil económico-digital de los estudiantes universitarios en tiempos de COVID-19. Desafíos, 12(1); 58-63. https://doi.org/10.37711/desafios.2021.12.1.280 
Copyright (c) 2022 Gema María Loor Ramos, Vicenta Inmaculada Aveiga Macay y Walter Zambrano Romero

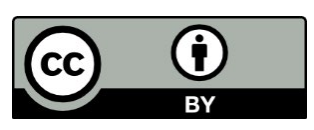

Este texto está protegido bajo una licencia internacional Creative Commons 4.0.

Usted es libre para Compartir-copiar y redistribuir el material en cualquier medio o formato

- y Adaptar el documento - remezclar, transformar y crear a partir del material-para cualquier propósito, incluso para fines comerciales, siempre que cumpla las condiciones de Atribución. Usted debe dar crédito a la obra original de manera adecuada, proporcionar un enlace a la licencia, e indicar si se han realizado cambios. Puede hacerlo en cualquier forma razonable, pero no de forma tal que sugiera que tiene el apoyo del licenciante o lo recibe por el uso que hace de la obra.

$\underline{\text { Resumen de licencia - Texto completo de la licencia }}$ 